\title{
Reflexiones sobre lo destituyente, los movimientos y el poder popular ¿Hacia nuevas formas de subjetivación?
}

\section{Some reflections on social movements, popular power and the destituyente capacity. Towards new subjectivity forms?}

\author{
Tomás Torres López ${ }^{1}$
}

En los procesos recientes latinoamericanos hemos vivenciado diferentes momentos de rupturas, algunos marcados fuertemente por apertura de caminos y otros de clausuras a los momentos de desarrollo de los sujetos populares. Las diversas experiencias ganadas en los últimos años dan cuenta de una impronta dialéctica clave en la lectura de dichos momentos, puesto que la formulación, crecimiento y consolidación de movimientos populares ha marcado un ciclo político con características propias de reapropiación y emergencia de una subjetividad colectiva dispuesta a marcar nodos de inflexión en la historia reciente de nuestro subcontinente.

En el presente ensayo buscamos abrir puntos de reflexión críticos sobre como comprender el proceso contradictorio entre momentos destituyentes y constituyentes en América Latina, esto como telón de fondo para comprender los nuevos sujetos políticos emergentes desde una subjetividad popular que se ha forjado en los últimos años por una imbricación de diferentes matrices sociopolíticas que dan cuenta de formas de cristalización de las clases subalternas en instituciones propias. Estas las denominaremos poder popular. En definitiva buscamos aportar a la reflexión crítica de cómo las características destituyentes de los movimientos populares pueden devenir formas concretas del ejercicio del poder popular.

\footnotetext{
${ }^{1}$ Chileno, Licenciado en Sociología por la Universidad de Viña del Mar y Magíster C e en Sociología por la Universidad Alberto Hurtado; dedicado al estudio de la sociología política, sociología de los movimientos sociales, marxismo y América Latina. E-mail:tom.torres.lopez@gmail.com
} 
No buscamos centrarnos en un país en específico, lo que daría cuenta de gramáticas propias en cada contexto, sino que contribuir a un debate necesario sobre el futuro de la acción colectiva en América Latina aportando reflexiones necesarias e impostergables sobre lo destituyente, el poder popular y las matrices sociopolíticas.

Finalmente, dado el contexto Latinoamericano actual hay diferentes interrogantes que guían el presente ensayo tales como ¿lo destituyente es un momento del cambio? ¿Existe una relación entre lo destituyente y el poder popular? ¿Cómo comprender la emergencia de nuevos sujetos políticos entre una dialéctica de instituir y destituir?

\section{Movimientos populares ¿Nuevas emergencias de sujetos políticos con viejos problemas?}

Los movimientos sociales, como cualquier otro tipo de acción colectiva, no están por fuera de la división clasista de la sociedad. Latinoamérica, particularmente, tiene diferenciaciones sociales muy marcadas, como también muy diversas. En este sentido es que preferimos utilizar el concepto de movimientos populares, ya que como menciona Camacho $(1987,: 9)$,

Podemos decir entonces que los movimientos sociales tienen dos grandes manifestaciones: por un lado, aquellos que expresan los intereses de los grupos hegemónicos y, por otro, los que expresan los intereses de los grupos populares. Estos últimos son los que conocemos como movimientos populares.

En este sentido los movimientos sociales son comprendidos como categorías amplias que no necesariamente expresan una proyección clasista de los sectores oprimidos, sino que estos tienen una dinámica de cuestionamiento fragmentado o absoluto [esto dependerá del momento constitutivo del movimiento popular (Ibíd. :10)] a las estructuras de dominación. Esto indica dos elementos, el primero que el nivel de cuestionamiento dependerá de la composición de clase y segundo que la característica fragmentaria o total a la dominación también tiene relación con las clases sociales.

Puesta en perspectiva las cosas consideramos que las clases y los movimientos sociales tienen una relación muy íntima, de importancia central para establecer conjeturas tanto entre los diferentes sistemas de estratificación social como también con la forma Estado en las 
diferentes regiones de nuestro continente. Posteriormente ahondaremos en las problemáticas teóricas para definir "lo" popular como un elemento objetivable de las experiencias subalternas.

Los movimientos populares pueden tener diferentes formas de actuar, si bien no compartimos tipologías de movimientos sociales, en el sentido de objetivar definiciones que por su desarrollo histórico son difíciles de delinear por su constante cambio y por la capacidad de agencia de los sujetos, creemos necesario mencionar la visión de totalidad en las luchas y perspectivas como punto de inicio para la discusión de las matrices. En este sentido consideramos necesario recalcar tres puntos que diferencian formas de acción colectiva. El comportamiento colectivo, comprendido como la restauración de algún elemento "enfermo" de la sociedad; las luchas sociales, como cambios introducidos en el campo político y el movimiento social, que es definido como un conflicto que persigue cambiar las relaciones de poder (Touraine, 1984). Podemos observar claramente una diferenciación, que a nuestro parecer nubla el diálogo importante sobre los movimientos como elementos heurísticos de la sociedad, pero a pesar de ello también aporta a entender los repertorios de acción y por ende su relación con las matrices.

Realizada esta breve explicación sobre las implicancias de pensar los movimientos populares y su relación con las matrices saltan a la luz los problemas en el actual contexto Latinoamericano. El primero es comprender la relación que hay entre gobiernos progresistas y movimientos populares, el segundo es entender la relación que se entabla entre la forma Estado (con las características actuales en los contextos de globalización) y los movimientos. La primera, tiene una doble dificultad (a) la reducción del conflicto al binarismo cooptaciónautonomía. Por otro lado, (b) la potenciación de ciertos conflictos por sobre otros y por ende perder el rumbo de los planteamientos iniciales que les permitieron establecerse como gobierno. Un ejemplo de esto es lo que ocurre con el Estado plurinacional boliviano, donde la matriz indigenista se entrecruza con la clasista lo que genera ciertas tensiones entre grupos subalternos por la hegemonía en relación a la agenda que debe adoptar el gobierno o la imposición de una respuesta autonómica a la implementación "occidentalizante" del Estado Boliviano. Esto ha llevado a un abierto reconocimiento de situaciones de tensión entre el Estado y el campo popular (Rauber, 2012). 
Consideremos dos puntos, el primero es que los movimientos sociales desde el sur, tienen una particularidad que los enmarca dentro de una tipología diferente a los de los países centrales, donde tanto la clausura operacional, en términos luhmanianos, es diferente como también la composición y las propuestas de cambio (de Sousa Santos, 2001) que estos tienen inherente en su formación, que si bien no es típicamente trabajadora-asalariada-obrera, contiene diferentes actores que los componen bajo un nueva perspectiva (Seoane, Taddei e Algranati, 2006) donde las demandas, emanadas en el último tiempo como eje aglutinador ha sido cambios estructurales en las formas democráticas, por lo menos en las últimas dos décadas (Borón, 2006; Modonesi, 2008). Las características mencionadas repercuten en pensar una manera de comprender los movimientos sociales desde el sur, con una mirada un tanto crítica, en su composición, pero también, y particularmente, en su producción de saberes, organizaciones, valores y apuestas de largo plazo en términos políticos.

Las características descritas, nos entregan dos elementos que nos parecen son importantes para el presente ensayo, el primero dice relación con que los movimientos populares tienen la característica de poseer un elemento clasista en su contenido, pero que no acaba en una visión económica de ella, por ende tiene características que contornean las posibilidades de exigencia y de repertorio político. Además, las características propias que adquieren en nuestro continente las acciones colectivas, demuestran que las formas de formulación de los sujetos políticos en nuestro continente obedecen a imbricaciones que permiten la apertura entre posibilidades concretas y cambio de orden.

\section{El carácter destituyente de los movimientos populares ¿Cambio de orden, nuevas propuestas?}

La férocité ancienne tend à être remplacée par la ruse et beaucoup de sociologues estiment que c'est là un progrès sérieux (...) tandis que nous voyons regner aujourd'hui le mensonge, le fausseté, la perfidie (G. Sorel, Reflexions sur la violence)

La cita de Sorel presenta un problema central para comprender el carácter destituyente y este tiene que ver no sólo con la violencia, que es el centro de su reflexión en el texto, sino que la producción discursiva que produce una subjetividad marcada por un encuentro entre el Estado y la sociedad civil. 
Antes de continuar es necesario realizar unas breves aclaraciones necesarias. La primera es comprender lo destituyente desde una perspectiva más amplia que el derrocamiento de gobiernos, es decir, no podemos reducir la discusión a cómo los movimientos populares son capaces de derrocar determinados regímenes. El segundo elemento, es comprender que el orden sobre el cual se fundan los movimientos populares dice relación con la confección y consolidación de un determinado tipo de subjetividad, que una vez que es superada, permite la articulación efectiva entre dos elementos: la experiencia y la utopía. Finalmente, emergencia de un orden latente permite comprender que lo destituyente no está exento de la institución de nuevas formas de vida.

La característica de lo destituyente ha caído en un lugar común para designar el cambio de mando de un tipo de gobernabilidad a otro (Modonesi, 2008; Svampa, 2008; Wilhelmi, s. f.). El tránsito del "giro progresista" en América Latina (Lanzaro, 2007) ha sido denominado como un cambio de época (Svampa, 2008) en el cual la formulación de los movimientos populares ha sido fundamental, puesto que el cambio de orden, desde una gobernanza neoliberal a una de corte progresista, ha marcado un camino que ha sido recorrido por diferentes países.

Consideramos que el poder destituyente, tiene una formulación que es más abarcadora que esta. Si la propuesta de Walter Bejamin (1998) en relación a la violencia mítica y divina podremos encontrar puntos que permiten pensar la formulación de las clases subalternas en relación al orden y la violencia como un complejo entramado de significantes que dan a conocer una nueva forma de entender la búsqueda de una alternativa que permita fundar una nueva sociabilidad. En efecto, si presuponemos, siguiendo al autor mencionado, que el derecho se funda en la violencia y que esta es inherente a las prácticas de la lucha de clases, entonces consideramos que la forma de poder superar la violencia originaria está marcada por la creación/producción de nuevas formas de vida.

En este sentido, la formulación destituyente tiene relación a cómo fundar un nuevo orden que supere en términos efectivos, el orden neoliberal. El cambio de gobernanza es sólo un momento de lo destituyente que aún no permite pasar de la negación a la afirmación, es decir, si bien el hecho de destituir a gobiernos neoliberales en el proceso mismo los movimientos y sus propuestas han sido apropiados por formas que cristalizan en términos constituyentes, 
nuevas constituciones de por medio, un orden que no deja de superar los escollos del anterior, vale decir, lo nuevo que no termina de nacer y lo viejo que no termina de morir.

Consideramos que al igual que la violencia no violenta de la creación de un nuevo orden (Benjamin, 1998) - que no es tal por el hecho de que no interrumpe el orden propio del primer término, sino que funda algo nuevo - lo destituyente tiene esa característica, es decir, la formulación de una nueva forma de comprender las relaciones sociales. Si reducimos el término sólo a un proceso de cambio de gobierno estamos dejando la parte creativa de los movimientos populares dejando de lado la posibilidad de instituir nuevas formas de relaciones sociales.

Creemos que las características destituyentes de los movimientos sociales tienen la capacidad de consolidar un proceso que supere el orden jurídico-político-económico en su totalidad, no parcialmente, por ende la apertura de posibilidades de generación de puntos de encuentro entre un nuevo contexto Latinoamericano y una profundización de las apuestas políticas mismas de los actores colectivos. Por ende, considerar lo destituyente sólo como un proceso de cambio de gobierno, aunque como lo hemos definido lo contenga, no es suficiente para definir un cambio de orden propiamente tal, ya que se han generado nuevos consensos (Quiroga, Canzani, Ensignia e Repetto, 2009) que si bien son proclives a los cambios estos comienzan a develar sus puntos límites (Zibechi, 2011).

Por otro lado, la capacidad destituyente dice relación con las formas que adquiere una determinada subjetividad política. Siguiendo el esquema propuesto por Modonesi (2010) podemos diferenciar tres formaciones subjetivas de lucha entre las clases subalternas y las dominantes. Estos elementos están basados en la experiencia, comprendida como una vía de síntesis entre las vivencias cotidianas y la formación efectiva de sujetos políticos, que, comprendiendo contextos de resistencia, lucha y emancipación producen actores colectivos capaces de disputar hegemonía e instaurar un nuevo orden. Cabe destacar que estas tres formas, que por el autor son definidas como, subalternidad, antagonismo y autonomía pueden combinarse, es decir, no existe una forma pura de uno de los "estados" sino que una imbricación cotidiana que da cuerpo a los contextos de lucha de clases. 
Desde esta perspectiva consideramos, que si bien hay una superposición entre estas tres formas de subjetivación, dependiendo del contexto y la formación de los movimientos populares una de ellas predominará por sobre las otras. En este sentido, creemos que el antagonismo, comprendido como la forma de lucha directa entre las clases dominantes y subalternas cristalizada en formaciones nacientes de nuevas relaciones sociales (Negri, 2004) predomina en lo que denominaremos subjetivación destituyente. Esta tiene características de formulación de nuevos consensos y apertura de posibilidades de cambio de orden profundo en nuestras sociedades.

Esta breve discusión permite dar luces sobre los procesos recientes en nuestro continente pero también sobre la posibilidad de tener una postura crítica sobre los procesos de avanzada de gobiernos progresistas, cuestión que es muy debatida desde la intelectualidad crítica de nuestro continente.

Finalmente, no queremos dejar de mencionar que la subjetivación destituyente no quiere decir que no exista capacidad de formular nuevas instituciones, es decir, no implica formaciones de organizaciones propias, es más, la subjetivación destituyente implica como condición necesaria la organización de movimientos populares para poder lograr llegar a este tipo de interacción contrahegemónica. Por ende continuaremos describiendo una de las posibilidades de configuración entre estas formas de subjetivación y una posible vía.

\section{El poder popular: posibilidades sociopolíticas del campo popular}

Es necesario mencionar que no hay una sola forma de concebir el poder popular, la multiciplicidad de prácticas remite también a la multiplicidad de sujetos que componen lo que denominamos campo popular, siguiendo a Falero ( s. f., p: 1):

En términos muy generales propongo denominar campo popular a ese arco o abanico de organizaciones y movimientos sociales proclives a la emancipación o transformación social existente en toda sociedad. En ese arco no incluyo partidos políticos ya que tienen su propia lógica de funcionamiento. 
En este sentido utilizar la lógica del campo permite comprender que es un espacio de tensión, luchas y disputas que están mediados por partidos, movimientos, ideologías y subjetividades que moldean y generan un tipo de habitus particular.

Sin adentrarnos en esta discusión queríamos dejar en claro desde donde nos posicionamos para comenzar a pensar el campo popular. Sin embargo, el segundo término es conflictivo para ser definido y utilizado. Muchas veces se ha mencionado que lo popular es igual a un pueblo, no obstante, esta definición no aporta a disipar las dudas y las dificultades de definición del concepto (Badiou et al., 2014) En este sentido la dificultad de definir en términos teóricos el concepto es a la vez la potencia real en la construcción social de significados y luchas concretas.

Lo popular lo comprendemos como un conjunto de sectores sociales que vivencian, es decir, experimentan la explotación y la dominación. Donde una se refiere al campo económico y la otra al ideológico (Camacho, 1987). Esto pareciera generar una diferencia con las tradiciones de izquierda en América Latina, puesto que como hemos mencionado se tendía a simplificar la discusión del sujeto copiando los esquemas traídos desde otras latitudes.

Esta vivencia hace referencia a la cultura, al ethos generador de saberes y prácticas que producen lo que hemos denominado subjetividades colectivas. Por esto comprendemos que:

El pueblo abarca también a todos aquellos sectores en cuanto se identifican y han hecho una opción por y junto a los sectores explotados, dominados y excluidos. Se trata entonces de un bloque ético-politico-social alternativo conformado entorno a un proyecto de liberación (Rebellato, 1995: 159.)

Lo anterior es una idea que plasma la identidad potencial que, mediados por una subjetividad política particular, desarrollan una praxis en conformación de sectores, por lo cual podríamos decir que el pueblo es un devenir sujeto político. Por otro lado, la composición social de lo que denominamos popular la definiremos como un conjunto de patrones, valores de las clases subalternas producidos en el contexto de los antagonismos principales, desde una máxima estructurante, los que viven de su trabajo y los que viven del trabajo ajeno (Mazzeo, 2014). No obstante, esto genera diferentes diversos procesos de apropiación, de lenguajes, símbolos, creencias, valores, modos de vida etc. Consideramos que la contradicción capital-trabajo es la base del capitalismo, sin embargo, este va aumentado día a día diversas mediaciones. 
Por otro lado, el peso del capitalismo industrial en nuestro continente nunca tuvo el mismo peso que en Europa (ibíd.) El peso de la fábrica, como lugar disciplinario, nunca fue el centro de la construcción socio-política de nuestras naciones, donde si bien es innegable el carácter capitalista de la producción la contradicción entre producción social y apropiación individual no sólo se presenta, en nuestro continente, entre burgueses y proletarios (ibíd.).

Comprenderemos por poder popular: "la autoorganización de las masas y la capacidad de control de éstas sobre la gestión de la sociedad" (Gaudichaud, 2004: 28). Es decir, la capacidad de auto-organización del pueblo con características instituyentes de soberanía. (Acha et al., 2007). Este proceso implica un nuevo Estado, movimientos populares con características instituyentes y una democracia radical (Rauber, 1994).

En este sentido encontramos dos características fundamentales que permiten el desarrollo de la constitución de subjetividades colectivas en el poder popular. La memoria salta a la luz cuando en la historia reciente de nuestro continente se desarrollaron diferentes procesos revolucionarios, con diversas características, como también la utopía el proyecto de autogobierno, sumado a que lo popular hace alusión a las experiencias de clase (Thompson, 1989) que permiten anclar en las clases sociales a los movimientos populares. Queremos recalcar que esta es una vía, que se debe discutir como todas las posibilidades, pero que tiene una particularidad, esta es que posee características propias, nuestras.

Las relaciones entre lo destituyente y el poder popular son claras, puesto que la formulación de un cambio de orden total al actual implica nuevas formas relacionamiento entre los sectores subalternos, pero también formas de instituir prácticas sociales que superen las subjetivaciones del capitalismo actual. Esto implica una dialéctica entre lo destituyente y el poder popular que está basada en formaciones concretas que puedan superar los momentos de desencuentro entre posibilidades y utopía (Darling, 2013).

\section{REFLEXIONES FINALES}

Las implicancias que hay entre la capacidad destituyente de los movimientos populares y el poder popular son claras y bastas, pero no del todo exploradas, ya que la atención se ha centrado en otros aspectos como las relaciones que se establecen entre gobiernos progresistas y actores colectivos, no obstante, la emergencia de subjetividades políticas antagonistas es 
sólo un tema tangencial, a pesar de la importancia que han ganado las alternativas emergentes y en construcción de los sujetos colectivos de nuestro continente.

El poder destituyente es un cuerpo teórico que requiere de una exploración mayor a la actual, puesto que si bien hemos dado un puntapié inicial, es sólo eso, la punta del iceberg de un concepto que puede dar cuenta, en buena medida, de los diversos procesos que actualmente vive nuestro continente. En la medida en que se articulen sectores subalternos con proyecto político se pueden dar cuenta de ejercicios analítico-prácticos que puedan abrir nuevas posibilidades de investigación.

\title{
RESUMEN
}

En el presente ensayo buscamos contribuir a una reflexión relativamente reciente que se ha dado en nuestro continente, a saber, la relación entre la capacidad destituyente de los movimientos populares y el poder popular. La necesidad de pensar en relación a estos tres términos es debido al actual escenario Latinoamericano. Las posibilidades de encuentro y desencuentro, como también vías para comprender nuevos espacios de subjetivación.

Palabras clave: Movimientos populares, capacidad destituyente, poder popular.

\begin{abstract}
In this paper we try to contributie to a recent reflextions in our continent. The posibility of the destituyente capacity of popular movements and popular power. The necesity of think in this therms is because de actual scenario requires the effort. The posibilitys of relation and the new spaces of subjectivation is an imperative.
\end{abstract}

Key words: popular movements, destituyente capacity, popular poder.

\section{REFERÊNCIAS BIBLIOGRÁFICAS}

ACHA, Omar et al. (2007), Reflexiones sobre el Poder Popular. Buenos Aires, Editorial El Colectivo.

BENJAMIN, Walter. (1998), Para una Crítica de la Violencia y otros Ensayos. Iluminaciones

IV. Introducción y selección de Eduardo Subirats. Traducción de Roberto Blatt. Madrid, Taurus Ediciones. 
BADIOU, Alan et al. (2014), ¿Qué es un Pueblo? Santiago, LOM.

BORÓN, Atilio. (2006), "Crisis de las Democracias y Movimientos Sociales en América Latina: Notas para una Discusión". OSAL, n 7, pp. 289-304.

CAMACHO, Daniel. (1987), "Movimientos Sociales, algunas Definiciones Conceptuales". Revista de las Ciencias Sociales, $\mathrm{n}^{\circ}$ 37-38, pp. 7-21.

DARLING, Victoria. (2013), "Reflexiones sobre el Poder Destituyente de los Movimientos Sociales en América Latina". Recuperado a partir de http://repositoriodigital.academica.mx/jspui/handle/987654321/188383

DE SOUSA SANTOS, Boaventura. (2001), "Los Nuevos Movimientos Sociales". OSAL, ${ }^{\circ}$ 5, pp. 177-188.

FALERO, Alfredo. (s. f.), Movimientos Sociales y la Recreacion del Campo Popular en Uruguay: Desafios Urgentes.

GAUDICHAUD, Franck. (2004), Poder Popular y Cordones Industriales: Testimonios sobre el Movimiento Popular Urbano, 1970-1973. Santiago, Lom Ediciones.

LANZARO, Jorge. (2007), "La 'Tercera Ola' de las Izquierdas Latinoamericanas: Entre el Populismo y la Social-democracia". Encuentros Latinoamericanos, vol. 1, nº 1, pp. 20-57. MAZZEO, Miguel. (2014), "El Sueño de una Cosa: Introducción al Poder Popular" (Segunda Edición). Tiempos Robados Editoras.

MODONESI, Massimo. (2008), "Crisis Hegemónica y Movimientos Antagonistas en América Latina. Una Lectura Gramsciana del Cambio de Época". A Contracorriente: Revista de Historia Social y Literatura en América Latina, vol. 5, nº 2, pp. 115-140. . (2010), Subalternidad, Antagonismo, Autonomía: Marxismos y Subjetivación

Política. Buenos Aires, CLACSO/ Prometeo Libros.

NEGRI, Antonio. (2004), Los Libros de la Autonomía Obrera (Vol. 25). Madrid, Ediciones AKAL.

QUIROGA, Yesko; CANZANI, Agustín; ENSIGNIA, Jaime. (2009). Consenso Progresista:

Las Políticas Sociales de los Gobiernos Progresistas del Cono Sur. Friedrich-Ebert-

Stiftung, Red de Centros y Fundaciones Progresistas del Cono Sur.

RAUBER, Isabel. (1994), Construyendo Poder desde Abajo. Santo Domingo,

Debate Popular. 
. (2012), Revoluciones desde Abajo: Gobiernos Populares y Cambio Social en

Latinoamérica. Buenos Aires, Peña Lillo

REBELLATO, José Luis. (1995), La Encrucijada de la Ética. Neoliberalismo Conflicto

Norte-sur. Montevideo, Nordan.

SEOANE, José; TADDEI, Emilio; ALGRANATI, Clara. (2006), "Las Nuevas

Configuraciones de los Movimientos Populares en América Latina", in A. Boron y G.

Lechini, Política y Movimientos Sociales en un Mundo Hegemónico. Lecciones desde

África, Asia y América Latina. Buenos Aires, CLACSO, pp. 227-250.

SVAMPA, Maristella. (2008), Cambio de Época: Movimientos Sociales y Poder Político.

Buenos Aires, Siglo XXI.

THOMPSON, Edward P. (1989), La Formación de la Clase Obrera en Inglaterra. Madrid,

Capitan Swing.

TOURAINE, Alain. (1984), "Social Movements: Special Area or Central Problem in

Sociological Analysis?" Thesis Eleven, vol. 9, n 1, pp. 5-15.

WILHELMI, Marco Aparicio. (s. f.). De la Fuerza Destituyente al Poder Constituyente:

Alcances de las Transformaciones Constitucionales en Ecuador y Bolivia.

ZIBECHI, Raúl. (2011), "Políticas Sociales, Gobiernos Progresistas y Movimientos

Antisistémicos". Otra Economía, vol. 4, nº 6, pp. 32-42.

Recebido em: 02/02/2016

Aprovado em: 09/03/2016 